\title{
Braconidae (Hymenoptera) in the collection of the Institute of Zoology, NAS of Azerbaijan Republic Part III. Subfamilies Helconinae, Brachistinae, Euphorinae, Macrocentrinae (Hymenoptera)
}

With 1 map

\author{
Arifa Abdinbekova ${ }^{1}$, Ellada Huseynova ${ }^{1,2}$ and llhama Kerimova ${ }^{1,3}$ \\ ${ }^{1}$ Institute of Zoology, NAS of Azerbaijan Republic, Az 1073, pr. 1128, bl. 504, Baku, Azerbaijan. - ${ }^{2}$ ella_line@mail.ru; \\ ${ }^{3}$ ilkershah@hotmail.com \\ Published on 2013-12-20
}

\section{Summary}

The paper gives some faunistic records of the braconids from genera Cenocoelius, Diospilus, Taphaeus belonging to subfamily Helconinae, from genera Eubazus, Polydegmon, Triaspis, Schizoprymnus belonging to subfamily Brachistinae, from genera Meteorus, Zele, Chrysopophthorus, Aridelus, Blacus, Dinocampus, Microctonus, Pigostolus, Centistes, Ancylocentrus, Leiphron, Peristenus, Wesmaelia, Syntretus belonging to subfamily Euphorinae and from genus Macrocentrus belongin to subfamily Macrocentrinae from the collection of the institute of Zoology of the National Academy of Sciences of Azerbaijan located in Baku. For each taxon localities in Azerbaijan, distribution and hosts remarks are given. 27 species from the collection is located in the ZIN (Zoological Institute of the RAS) so we have not presented information about them. This concerns: Centris (Ancylocentrus) subculcatus (Thomson, 1895), Allurus muricatus (Haliday, 1833), Eubazus (Aliolus) kusarensis (Aвdinbekova,1969), Eubazus (Allodorus) semirugosus (Nees, 1816), Chrysopophthorus hungaricus (KIss, 1927), Ecclitura primoris KoKuyev, 1902, Eubazus (Eubazus) pallipes NeEs, 1816, E. (Brachistes) rufithorax (AвDInBeKova, 1969), E. (Brachistes) minutus (RATzeBURg, 1848), E. (Brachistes) talitzkii Tobias, 1976, Schizoprymnus crassiceps (Thomson, 1891), Schizoprymnus elongatus (SzÉPligeti, 1898), Sch. subangustatus Tовias 1976, Sch. ambiguus (NeEs, 1816), Sch. globosus (SzÉPligeti, 1898), Helcon tardator Nees, 1814, Homolobus infimator (Lyle, 1914), Homolobus annulicornis (NeEs, 1834), Leiophron palidistigma CurTis, 1833, Meteorus heliophilus Fischer, 1970, Meteorus cespitator (Thunberg, 1822), Perilitus (Townesilitus) bicolor (Wesmael 1835), Perilitus (Townesilitus) breviradialis (ToBIAs, 1976), Macrocentrus brevicaudifer VAN AcHTERBERg 1979, Macrocentrus flavus Snellen van Vollenhoven, 1878, Macrocentrus thoracicus (NeEs, 1812), Macrocentrus marginatus (NeEs, 1811).

\section{Zusammenfassung}

In der Arbeit werden faunistische Daten der Braconiden-Gattungen Cenocoelius, Diospilus, Taphaeus (Unterfamilie Helconinae), Eubazus, Polydegmon, Triaspis, Schizoprymnus (Unterfamilie Brachistinae), Meteorus, Zele, Chrysopophthorus, Aridelus, Blacus, Dinocampus, Microctonus, Pigostolus, Centistes, Ancylocentrus, Leiphron, Peristenus, Wesmaelia, Syntretus (Unterfamilie Euphorinae) und Macrocentrus (Unterfamilie Macrocentrinae) des Sammlungsmaterials aus dem Institut für Zoologie der nationalen Akademie der Wissenschaften von Aserbaidschan in Baku ausgewertet. $\mathrm{Zu}$ jedem Taxon werden Fundorte in Aserbaidschan, Verbreitung und Wirte genannt. Von 27 Arten aus der Sammlung des ZIN (Zoologisches Institut der Russischen Akademie der Wissenschaften) fehlen diese Informationen jedoch. Das betrifft: Centris (Ancylocentrus) subculcatus (Thomson, 1895), Allurus muricatus (Haliday, 1833), Eubazus (Aliolus) 
kusarensis (АвDInbeкova, 1969), Eubazus (Allodorus) semirugosus (NeEs, 1816), Chrysopophthorus hungaricus (KIss, 1927), Ecclitura primoris KoKuyev, 1902, Eubazus (Eubazus) pallipes Nees, 1816, E. (Brachistes) rufithorax (AbDinbekova, 1969), E. (Brachistes) minutus (Ratzeburg, 1848), E. (Brachistes) talitzkii ToBias, 1976, Schizoprymnus crassiceps (Thомson, 1891), Schizoprymnus elongatus (SzÉPligeti, 1898), Sch. subangustatus ToвiAs 1976, Sch. ambiguus (NeEs, 1816), Sch. globosus (SzÉPligeti, 1898), Helcon tardator NeEs 1814, Homolobus infimator (Lyle, 1914), Homolobus annulicornis (NeEs, 1834), Leiophron palidistigma Curtis, 1833 Meteorus Heliophilus Fischer, 1970, Meteorus cespitator (Thunberg, 1822), Perilitus (Townesilitus) bicolor (Wesmael 1835), Perilitus (Townesilitus) breviradialis (Tobias, 1976), Macrocentrus brevicaudifer van Achterberg 1979, Macrocentrus flavus SNELLEN van VolLENHOVEN, 1878, Macrocentrus thoracicus (NeEs, 1812), Macrocentrus marginatus (NeEs, 1811).

\section{Introduction}

Subfamily Helconinae is presented by 3 species from 3 genera, subfamily Brachistinae by 17 species from 4 genera and subfamily Euphorinae by 28 species from 12 genera, subfamily Macrocentrinae by 4 species from 1 genus in the entomological collection of the Institute of Zoology NAS of Azerbaijan. The paper includes distribution remarks and host range information for each species. The whole distribution areas in the world are provided for each taxon. Each name of species is accompanied by the author's name and description date. A map showing collection areas in Azerbaijan is presented. Literature data are used for taxonomic analysis of the braconids (ABDINBEKova, 1969, 1995; PAPP, 2007, 2009; ToBIAs, BELOKoBYlskij, Kotenko, 1995, Shenefelt, 1970, Huddleston, 1980, Lozan, 2004, Van A. Achterberg, 1988, 2003).

\section{Subfamily HELCONINAE}

Genus Cenocoelius Westwo0d, 1840

Cenocoelius analis (NeEs, 1834)

Material: dist. Ordubad, 2.VI.1967, 1 ○َ, leg. Abdinbekova.

\section{Hosts:}

Magdalis armigera Geof., M. ruficornis L., Scolytus rugulosus P.W. J. MüL. (Curculionidae), Tetrops praeusta L. (Cerambycidae).

\section{Distribution:}

West Europe, Azerbaijan (AвDinbekova, 1995).

Genus Diospilus HaLIDAY, 1833

Diospilus rufipes (REINHARD, 1862)

Material:

dist. Guba, 14.V.1962, 1 ơ , leg. Abdinbekova.

\section{Hosts:}

Xestobium plumbeum Ill. (Anobiidae), Byctiscus populi L. (Attelabidae), Tachyerges salicis L. (Curculionidae).

\section{Distribution:}

West Europe, Caucasus, Azerbaijan (Abdinbekova, 1995).

\section{Genus Taphaeus WesmaEL, 1835}

Taphaeus hiator (ThunBERG, 1822)

\section{Material:}

dist. Khachmaz, 30.V.1960, 1 ๙ $^{\star}$, leg. Abdinbekova; dist. Kalbajar, 30.VI.1960, 1 o , leg. Abdinbekova.

Host:

Orchesia micans Pz., (Melandryidae).

\section{Distribution:}

West Europe, Caucasus, Western Kazakhstan, East Siberia, Azerbaijan (Aвdinbekova, 1995).

\section{Subfamily BRACHISTINAE}

\section{Genus Eubazus Nees von EsenbeCK, 1814}

Subgenus Brachistes WeSMAEL, 1835

Eubazus (Brachistes) gallicus (ReInHARD, 1867)

\section{Material:}

dist. Lankaran, 20.V.1965, 1 ○َ, leg. Abdinbekova.

Host:

Megdalis ruficornis (L.) (Curculionidae).

\section{Distribution:}

Europe, Turkey (Beyarslan, 2011), Caucasus, Azerbaijan (AbDinbeKova, 1995). 
Eubazus (Brachistes) parvulus (RUTHE, 1867)

Material:

dist. Khachmaz, 28.IV.1963, 1 ơ , leg. Abdinbekova.

Host:

unknown.

\section{Distribution:}

Europe, Mongolia (PAPP, 2009), Caucasus, Azerbaijan (Abdinbekova, 1995).

Subgenus Eubazus NeES von EsenBeCK, 1814

Eubazus (Eubazus) rufiventris (ABDINBEKOVA, 1975)

\section{Material:}

dist. Khachmaz, 17.V.1962, 1 ơ , leg. Abdinbekova.

Host:

unknown.

Distribution:

Turkey, Caucasus, Azerbaijan (Abdinbekova, 1995).

\section{Eubazus (Eubazus) tibialis (HaLIDAY 1835)}

\section{Material:}

dist. Ordubad, 15.V.1965, 1 ơ , leg. Abdinbekova.

\section{Hosts:}

Byctiscus betulae L., B. populi L. (Attelabidae), Gilpinia pallida KL. (Diprionidae), Anobium sp. (Coleoptera, Anobiidae) (SHENEFELT, 1970).

\section{Distribution:}

West Europe, Turkey, Caucasus, Azerbaijan (Shenefelt, 1970; Tobias et al. 1995, ABdinbeKova, 1995).

Genus Polydegmon FöRSTER, 1862

\section{Polydegmon sinuatus FöRSTER, 1862}

\section{Material:}

dist. Ordubad, 20-29.V.1967, 11 q \&, $70^{\top} 0^{\top}$, dist. Julfa, 21.V.1967, 2 ㅇ ㅇ, leg. Abdinbekova.

Host:

unknown.

\section{Distribution:}

West Europe (Vladimir, Van Achterberg, 2010), Turkey, Kazakhstan, Uzbekstan, Caucasus, Azerbaijan (SHENefelt, 1970; Tobias et al. 1995, Abdinbekova, 1995).

\section{Genus Triaspis HaLIDAY, 1835}

Triaspis aciculatus (RATZEBURG, 1848)

Material:

dist. Guba, 23.IV.1963, 1 ๙ै$^{\text {}}$, leg. Abdinbekova; dist.

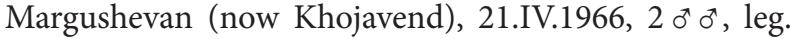
Abdinbekova.

Hosts:

Anobium rufipes F., Ochina ptinoides MarsH., Gastrallus corsicus ScHIL., (Anobiidae), Anthaxia quadripunctata (L.) (Buprestidae), Anthonomus rubi Herbst, Ceutorhynchus pleurostigma MARSH., Stenocarus ruficornis (STEPH.), Rhinusa antirrhini РАҮк., Gymnetron villosulum GylL. (Curculionidae), Apion damryi DesB. (Brentidae).

\section{Distribution:}

West Europe (GAvin et al. 2011), Caucasus, Azerbaijan (Abdinbekova, 1995, Tobias et al. 1995).

\section{Triaspis caucasicus AbDinBEKOVA, 1934}

\section{Material:}

dist. Marqushevan (now Khojavend), 20.V.1966, $120^{\top} 0^{\star}$, leg. Abdinbekova; dist. Lerik, 20.VI.1965, 8 우, leg. Abdinbekova; dist. Goygol, 23.V.1966, 1 ơ , leg. Abdinbekova; dist. Guba, 11.VI.1961, 1 o , leg. Abdinbekova.

\section{Host:}

unknown.

\section{Distribution:}

Turkey, Kazakhstan, Caucasus, Azerbaijan (AвDINBEKOVA, 1995, ToBias et al. 1995).

\section{Triaspis caudatus (NeEs von ESENBECK, 1816)}

\section{Material:}

dist. Ordubad, 1.VI.1967, 1 ๙ $^{\text {, }}$ leg. Abdinbekova; Nakhichevan AR, 19.VI.1967, 1 \%, leg. Abdinbekova.

Hosts:

Ochina ptinoides MARsh. (Anobiidae), Bruchidius fasciatus Ot. (Bruchidae), Byctiscus betulae (L.), Byctiscus populi (L.) (Attelabidae), Protapion assimile W. KIrBY, P. trifolii L., (Brentidae). 


\section{Distribution:}

Europe, Caucasus, Azerbaijan (AвDinbeKova, 1995, ToBiAs et al. 1995).

\section{Triaspis obscurellus (NEES von ESENBECK, 1816)}

\section{Material:}

dist. Guba, 12.V.1962, 2 ㅇ ㅇ, leg. Abdinbekova; dist. Khachmaz, 15.V.1963, 1 o , leg. Abdinbekova; dist. Mirbashir (now Tartar), 27.III.1966, 2 ㅇ 우 leg. Abdinbekova; Margushevan (now Khojavend), 29-31.III.1966, 4 우 우 leg. Abdinbekova; dist. Barda, 30.III.1966, $230^{*} \sigma^{*}$, leg. Abdinbekova.

\section{Hosts:}

Bruchus rufimanus (Bruchidae), Ceratapion carduorum Kву, (Brentidae), Ceutorhynchus assimilis PAYк., C. contractus Marsh., C. picitarsis Gyll., C. sulcicollis Gyll., C. pleurostigma MARsH., Gymnetron antirrhini PAYK., G. noctis HBst., Rhinusa collina, (Curculionidae) (SHENEFELT, 1970).

\section{Distribution:}

West Europe (GAvin et al. 2011), Greece (LozAN, 2004), Siberia, Mongolia, Caucasus, Azerbaijan (Shenefelt, 1970, ToвiAs et al. 1995, АвDinbekova, 1995).

\section{Triaspis pallipes (NEES von ESENBECK, 1816)}

Material:

dist. Guba, 14.V.1962, 1 ơ, leg. Abdinbekova.

\section{Hosts:}

Bruchidius seminarius L., Bruchus affinis F., B. atomarius L., B. lentis F., B. rufimanus, (Bruchidae), Anthonomus pomorum (L.), Ceutorhynchus assimilis (P.), Rynchaenus alni, R. quercus (L.), R. semirufus GyLl. (Curculionidae).

\section{Distribution:}

West Europe (GAvin et al. 2011), Caucasus, Azerbaijan (AвDinbekova, 1995, Tobias et al. 1995)

\section{Triaspis thoracicus (CURTIS, 1860)}

Material: dist. Yardimly, 20.VI.1965, 1 ơ , leg. Abdinbekova.

\section{Hosts:}

Acanthoscelides obtectus SAY, Bruchus rufimanus B., Bruchus pisorum (L.) (Bruchidae).

\section{Distribution:}

West Europe (GAvin et al. 2011), Caucasus, Azerbaijan (ABDinbeKova, 1995).

\section{Genus Schizoprymnus FöRSTER, 1862 \\ Schizoprymnus angustissimus ŜNŏFLAK, 1952}

\section{Material:}

dist. Kalbajar, 3.VI.1966, 1 ơ, leg. Abdinbekova; dist. Ordubad, 5.VI.1967, 3 o $\sigma^{\star}$, leg. Abdinbekova; dist. Mardakert (now Aghdere), 16.VI.1967, $10^{\star}$, leg. Abdinbekova.

\section{Host:}

unknown.

\section{Distribution:}

Former USSR, Check Republick, Kazakhstan, (SHENEFELT, 1970; Tobias et al. 1995), Turkey (GuClu, 2006), Caucasus, Azerbaijan (Aвdinbekova, 1995).

\section{Schizoprymnus azerbajdzhanicus ABDINBEKova, 1912}

Material:

dist. Barda, 30.III.1966, 1 ㅇ, leg. Abdinbekova; dist. Agdam, 11.IX.1964, 1 \% , leg. Abdinbekova; dist. Goychay, 3.VII.1964, 2 o $^{\star}$ o $^{\star}$, leg. Abdinbekova.

Host:

unknown.

\section{Distribution:}

Turkey (Guclu, 2006), Azerbaijan (Aвdinbekova, 1995).

\section{Schizoprymnus nigripes (THOMson, 1892)}

\section{Material:}

dist. Nukha (now Sheki), 27.V.1966, $1 \sigma^{\star}$, leg. Abdinbekova; dist. Ordubad, 29.V.1967, 1 ơ, leg. Abdinbekova.

Hosts:

Curculio rubidus Gyll., Acentrus histrio (FALD). (Curculionidae) (GUCLU, 2006).

\section{Distribution:}

Turkey (GuClu, 2006), Mongolia (PAPP, 2009), Caucasus, Azerbaijan (AвDINBEKova, 1995, ToвiAs et al.1995). 


\section{Schizoprymnus obscurus NeES von ESENBECK, 1813}

\section{Material:}

dist. Guba, 5.VIII.1961-1962, 5 ơ $\sigma^{\pi}$, leg. Abdinbekova; dist. Khachmaz, 5.VI.1962-1963, 5 o $^{\circ} \sigma^{\circ}$, leg. Abdinbekova; dist. Goychay, 7.VIII.1964, 3 우 웅, leg. Abdinbekova; dist. Lerik, 20.VI.1965, 1 ơ, leg. Abdinbekova; dist. Gasym-Ismayilov (now Goranboy), 14.VII.1966, 1 ㅇ, leg. Abdinbekova; dist. Mardakert (now Aghdere), 4.VII.1967, 3 ơ o o $^{\star}$ leg. Abdinbekova.

\section{Hosts:}

Ceratapion carduorum Kву (Brentidae), Ceuthorrhynchus picitarsis Gyll., C. sulcicollis Gyll., C. assimilis PAYK., Gymnetron noctis Hвsт., Rhinusa collina GyLL, Rh. antirrhini PAYK., (Curculionidae).

\section{Distribution:}

West Europe (GAvin et al. 2011), Turkey, (Guclu, 2006), Mongolia (PAPP, 2009), Former USSR, Caucasus, Azerbaijan (Aвdinbekova, 1995, Tobias et al. 1995).

\section{Schizoprymnus opacus (Thomson, 1892)}

\section{Material:}

dist. Agdam, 11.V.1964, 1 \%, leg. Abdinbekova; dist. Nukha (now Sheki), 27.V.1965, $10^{*}$, leg. Abdinbekova;

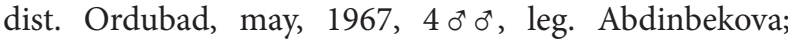
Nakhichevan AR, 17.VI.1967, $10^{*}$, leg. Abdinbekova; dist. Goygol, 18.VI.1967, 1 ơ , leg. Abdinbekova.

Host:

unknown.

\section{Distribution:}

West Europe, Mongolia, Former USSR (Shenefelt, 1970; Tobias et al. 1995), Caucasus, Azerbaijan (AbDinBEKOVA, 1995).

\section{Schizoprymnus terebralis ŜNŎFLAK, 1952}

\section{Material:}

dist. Agdam, 26.IV.1966, 1 o , leg. Abdinbekova; dist. Shahbuz, 22.V.1967, 1 \% , leg. Abdinbekova; dist. GasymIsmayilov (now Goranboy), 9.VI.1966, 1 \%, leg. Abdinbekova.

\section{Host:}

Mordellistena parvula GylL. (Mordelidae).

\section{Distribution:}

Check Republic, Kazakhstan (Shenefelt, 1970; Tobias et al. 1995) Mongolia (PAPP, 2009), Caucasus, Azerbaijan (Abdinbekova, 1995, Tobias et al. 1995).

\section{SUBFAMILY EUPHORINAE}

\section{Genus Aridelus MarshalL, 1887}

Aridelus egregius (SCHMIEDEKNECHT 1907)

\section{Material:}

dist. Kalbajar, 23.VII.1966, 1 (damaged), leg. Abdinbekova.

\section{Hosts:}

Aelia acuminata (L.), Aelia cognata FieB., Dolycoris baccarum (L.), Eurydema ornata (L.), Holcostethus vernalis Wolf., Palomena prasina (L.) (Pentatomidae), Coptosoma mucronatum SeID., Coptosoma scutellatum GeOFFr. (Plataspididae), Eurygaster austriaca Sснвк.), Eurygaster maura (L.), (Scutelleridae).

\section{Distribution:}

Turkey, Former USSR, Caucasus, Azerbaijan (ABDINBEKovA, 1995).

\section{Genus Blacus NeEs, 1817}

\section{Blacus armatulus RUTHE, 1861}

Material:

dist. Khachmaz, 6.VI.1963, 1 o , leg. Abdinbekova; dist. Mardakert (now Aghdere), 21.VI.1967, $10^{7}$, leg. Abdinbekova.

\section{Hosts:}

Parasite of Curculionidae (Coleoptera) (VAn AchterBERG, 1988).

\section{Distribution:}

Europe, Russia, Turkey, Moldova, Russia, Caucasus, Azerbaijan, Kazakhstan (ToвiAs, 1986, АвdinbeKova, 1995).

\section{Blacus exilis (NeEs 1811)}

Material:

dist. Khachmaz, 6.VI.1963, 1 (damaged), leg. Abdinbekova.

\section{Hosts:}

Pityokteines vorontzowi JACOBs., Magdalis armigera Geofrr. (Curculionidae), Scolytidae, Curculionidae, Anobiidae (Coleoptera).

\section{Distribution:}

Caucasus, Kazakhstan, Mid Asiya, Eastern Siberia, West Europe, Turkey, Israel, Mongolia, North America (Tobias, 1986). 


\section{Blacus instabilis RUTHE, 1861}

\section{Material:}

dist. Guba, 7.VII.1962, 1 ơ, leg. Abdinbekova.

Host:

unknown.

\section{Distribution:}

Ukraine, North Asia, West Europe, Turkey, Caucasus, Azerbaijan (Tobias, 1986, АвDinbeKova, 1995)

\section{Blacus ruficornis (NeEs 1811)}

Material:

dist. Gusar, 9-10.VII.1960, 3 ㅇ , leg. Abdinbekova; dist. Zaqatala, 12.VII.1960, 4 ठ $^{\text {ते }}$, leg. Abdinbekova; dist. Guba, 5.VI.1961-62, 7 우 우, leg. Abdinbekova; dist. Mirbashir (now Tartar), 5.VI.1966, 3 व $^{\text {त }}$, leg. Abdinbekova; dist. Gadabay, 28.VIII.1966, $50^{\top} 0^{\star}$, leg. Abdinbekova; dist. Khachmaz, 6.VII.1960-62, 1 ơ, leg. Abdinbekova; dist. Lankaran, 18.VIII.1964, 1 ơ, leg. Abdinbekova; dist. Lerik, 5.VII.1965-66, 2 (damaged), leg. Abdinbekova; dist. Gasym-Ismayilov (now Goranboy), 22.V.1966, $10^{7}$, leg. Abdinbekova; dist. Goygol, 24.IX.1966, $10^{\text {t, }}$ leg. Abdinbekova; dist. Shusha, 18.VIII.1966, 3 우 ㅇ, leg. Abdinbekova; dist. Mardakert (now Aghdere), VI.1966-1967, 3 đ $^{\text {त }}$, leg. Abdinbekova; dist. Dashkasan, 15.VII.1966, 1 o , leg. Abdinbekova; dist. Shamkhor (now Shamkir), 27.VII.1966, 1 ㅇ, leg. Abdinbekova; dist. Goychay, 19.V.1964, 1 †, leg. Abdinbekova.

\section{Host:}

Tachyporus obtusus L. (Staphylinidae)

\section{Distribution:}

West Europe, Russia, Caucasus, Azerbaijan, Kazakhstan, Siberia, North Asia, North Africa (ToBIAs, 1986, AbdinBEKOVA, 1995).

\section{Genus Centistes HaLIDAY, 1835}

\section{Subgenus Ancylocentrus}

Centistes (Ancylocentrus) edentatus (HaLIDAY, 1835)

Material:

dist. Gusar, 10.VII.1960, 1 ㅇ, leg. Abdinbekova; dist. Masally, 18.V.1961, 1 ơ, leg. Abdinbekova.

\section{Distribution:}

West Europe (GAvin et al. 2011), Kazakhstan (ToBiAs, 1986), Caucasus, Azerbaijan (AвDInBeKova, 1995).

Subgenus Centistes HaLIDAY, 1835

Centistes (Centistes) cuspidatus Haliday, 1833

\section{Material:}

dist. Gusar, 7-10.VII.1960, 4 ○ ठ Guba, 29.V.1962, 1 ㅇ, leg. Abdinbekova.

Hosts:

adults of the genus Tachyporus (Staphylinidae).

\section{Distribution:}

West Europe, Kazakhstan, Kirgizia, Sakhalin Islands, Azerbaijan (ToвiAs, 1986, АвDinbekova, 1995).

\section{Centistes (Centistes) fuscipes (NeEs, 1834)}

\section{Material:}

dist. Guba, 15.VI.1961, 1 đ̊ , leg. Abdinbekova; dist. Goranboy, 9.VI.1966, 1 ơ , leg. Abdinbekova.

\section{Host:}

Elachista trapeziella Sт. (Elachistidae).

\section{Distribution:}

West Europe, Sakhalin Islands, Primoryan territory (BelokовуцSкіJ, 1992), Caucasus, Azerbaijan (AbdinBEKOVA, 1995).

\section{Genus Chrysopophthorus GoIDANICH, 1948}

\section{Chrysopophthorus elegans ToBIAS, 1961}

\section{Material:}

Nakhichevan AR, 3.VIII.1964, 1 ơ, leg. Abdinbekova.

Host:

Chrysopa carnea ST. (Chrysopidae).

\section{Distribution:}

Azerbaijan (АвDINBEKova, 1995).

Host:

unknown. 
Genus Dinocampus Förster, 1862

Dinocampus coccinella (ScHRANK, 1802)

\section{Material:}

dist. Guba, 11.VI.1961, 1 ㅇ, leg. Abdinbekova; dist. Astara, 25.IV.1965, 1 ơ, leg. Abdinbekova; dist. Agdash, 8.VII.1966, 1 o , leg. Abdinbekova; dist. Mardakert (now Aghdere), 26.VI.1967, $10^{\star}$, leg. Abdinbekova; dist. Ordubad, 15.VI.1967, 1 ㅇ, leg. Abdinbekova; dist. Mirbashir (now Tartar), 16.V.1967, 1 ㅇ, leg. Abdinbekova; dist. Nukha (now Sheki), 28.V.1965, 1 ㅇ, leg. Abdinbekova; dist. Gakh, 24.VIII.1965, 1 ๙ , leg. Abdinbekova; dist. Khachmaz, 15.V.1963, 1 ๙ , leg. Abdinbekova.

Hosts:

Adalia bipunctata, A. decempunctata L., A. deficiens Muls., A. flavomaculata, Cheilomenes sexmaculata F., C. lunata F., Coccinella quinquepunctata L., C. californica Mann, C. trifasciata perplexa Muls., C. undecimpunctata aegyptiaca ReICHE., Harmonia axyridis PALL., Coelophora biplagiata (Sw), Coleomegilla maculata lengi Tiмв., Cryptolaemus montrouzieri Muls., Eriopis sede-cimpustulata LATr., Hippodamia convergens GuERIN, H. parenthesis SAY., Megilla maculata De GeEr. (Coccinellidae).

\section{Distribution:}

Europe, Japan, China, Egypt, Azerbaijan (Shenefelt, 1970, Toвias et al. 1995, AbDinbeKova, 1995).

Genus Leiophron NeEs, 1818

Subgenus Leiophron NeES, 1819

Leiophron (Leiophron) apicalis HALIDAY, 1833

\section{Material:}

dist. Guba, 29.V.1960, 1 ơ, leg. Abdinbekova.

Hosts:

Orthotylus adenocarpi Perr. Orthotylus artemisiae J. SH., Orthotylus beieri WAG., Orthotylus dimorphus WAG. (Miridae).

\section{Distribution:}

West Europe, Mid Asia, Ukraine, Kazakhstan, Azerbaijan (AbdinbeKova, 1995).

Subgenus Peristenus FöRSTER, 1862

Leiophron (Peristenus) reclinator (RUTHE, 1856)

\section{Material:}

dist. Khachmaz, 11.VIII.1962, 1 ๙ , leg. Abdinbekova; dist. Kirovabad (Ganja), 18.VI.1967, $20^{-1} o^{*}$, leg. Abdin- bekova; dist. Goranboy, 23.VI.1967, $1 \sigma^{\star}$, leg. Abdinbekova; dist. Julfa, 21.V.1967, 1 o , leg. Abdinbekova.

Hosts:

Lygocoris pabulinus L., Liocoris tripustulatus F. (Miridae).

\section{Distribution:}

Mid Europe, Azerbaijan (Toвias, 1986, Авdinbekova, 1995).

\section{Leiophron (Peristenus) orchesiae CURTIS, 1833}

\section{Material:}

dist. Masally, 18.V.1965, $4 \sigma^{\top} \sigma^{\top}$, leg. Abdinbekova; dist. Shamakhy, 27.V.1966, 1 ox, leg. Abdinbekova.

\section{Hosts:}

Closterotomus norwegicus (GML.) (Miridae), Orchesia micans PANz. (Melandryidae).

\section{Distribution:}

West Europe, Azerbaijan (Tobias, 1986, Aвdinbekova, 1995).

\section{Leiophron (Peristenus) pallipes CuRTIS, 1833}

\section{Material:}

dist. Guba, 5.VII.1961-1962, 4 o $^{\top}$, leg. Abdinbekova; Nakhichevan AR, 25.V.1967, 1 ㅇ, leg. Abdinbekova; dist. Kirovabad (now Ganja), 18.VI.1967, 1 o $^{\star}$, leg. Abdinbekova.

\section{Hosts:}

Adelphocoris lineolatus Gz., Adelphocoris rapidus SAY, Capsus ater (L.), Closterotomus norvegicus (GML.), Labops hirtus KNIGHT, Leptopterna dolabrata (L.), Lygus borealis (Kelt.), Lygus desertus Knight, Lygus elisus VAN D., Lygus hesperus KNIGHT., Lygus lineolaris (P. DE B.), Lygus pratensis (L.), Lygus rugulipennis Popp, Notostira erratica (L.), Plagiognathus medicagus AR., Trigonotylus coelestialium (KIRK.) (Miridae), Timarcha tenebricosa anglica BECH., Timarcha tenebricosa (F.) (Chrysomelidae), Eustrophus dermestoides (F.) (Tetratomidae).

\section{Distribution:}

Europe, Turkey (Yilmaz et al. 2008), Former USSR, Caucasus, Azerbaijan (ToBiAs, 1986, AвDinBekova, 1995). 
Leiophron (Peristenus) picipes CURTIS, 1833

\section{Material:}

dist. Lerik, 5.VII.1965, 2 우, leg. Abdinbekova; dist. Goygol, 24.IV.1966, 1 ơ , leg. Abdinbekova.

\section{Host:}

unknown.

\section{Distribution:}

Europe, Egypt, Chine (Shenefelt, 1970), Turkey (YIlMAZ, 2008), Former USSR, Caucasus, Azerbaijan (ABdinbeKova, 1995).

\section{Genus Meteorus HaLIDAY, 1835}

\section{Meteorus abdominator NeES von EsenBeCK, 1811}

\section{Material:}

dist. Guba, 18.VII.1961, 1 \%, leg. Abdinbekova; dist. Lerik, 20.VII.1965, 1 ơ , leg. Abdinbekova; dist. Dashkasan, 23.VIII.1966, $10^{\top}$, leg. Abdinbekova; dist. Gadabay, 1.VII.1967, 1 ơ, leg. Abdinbekova; dist. Shahbuz, 14.IX.1967, $10^{7}$, leg. Abdinbekova.

\section{Hosts:}

Cheimatobia brumata L., Eucosmia certata Hвn., Larentia fluctuate L., L. viridaria L., (Geometridae) (HuddLEston 1980), Gymnaetron spp. (Curculionidae) (GuCLu, 2006).

\section{Distribution:}

Europe (GAvin et al. 2011), Japan, Former USSR (HUdDLESTON 1980), Caucasus, Azerbaijan (АвDinbekova, 1995).

\section{Meteorus filator (HALIDAY, 1835)}

\section{Material:}

dist. Dashkesan, 7.VIII.1966, $2 \sigma^{\pi} \sigma^{\pi}$, leg. Abdinbekova.

Host:

unknown.

\section{Distribution:}

Europe, Turkey, Russia, Azerbaijan (ABDinbekova, 1995).

\section{Meteorus gyrator (ThunBERG, 1822)}

\section{Material:}

dist. Astara, 25.VI.1965, 1 o ${ }^{\star}$, leg. Abdinbekova.

\section{Hosts:}

Lacanobia oleracea (L.), Mamestra brassica (L.), Spodoptera exigua (Hвn.), Spodoptera littoralis (BoIsD.), Chrysodeixis chalcites (Esp.), Cosmia diffinis (L.) (Noctuidae).

\section{Distribution:}

Europe, Egypt, Russia, Azerbaijan (AвDinвeкova, 1995).

\section{Meteorus ictericus (NeEs, 1811)}

\section{Material:}

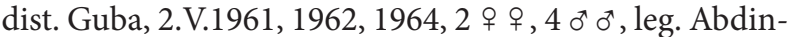
bekova.

Hosts:

Archips crataegana HвN., Archips oporana (L.), Archips rosana (L.), Archips xylosteana (L.), Tortrix viridana (L.) (Tortricidae), Apamea remissa(HвN.), Cosmia trapezina (L.), Euproctis chrysorrhoea(L.), Eupsilia transversa (HFN.), Helicoverpa armigera (HBN), Leucania loreyi (Dup.), Leucoma salicis (L.), Spodoptera exigua (HвN), Thaumetopoea pityocampa Den. \& SCHIF., Thaumetopoea processionea (L.) (Noctuidae), Abraxas grossulariata (L.), Erannis defoliaria (Cl.), Hemithea aestivaria (HвN.), Eupithecia tripunctaria (H.-S.), Eupithecia virgaureata DoubL., Operophtera brumata (L.), Rhoptria asperaria (HвN.), (Geometridae), Dendrolimus pini (L.) (Lasiocampidae).

\section{Distribution:}

Europe, Turkey, China, Japan, Korea, Azerbaijan (AbDINBEKOVA, 1995)

\section{Meteorus pulchricornis (WeSMAEL, 1835)}

\section{Material:}

dist. Ordubad, 29.V.1967, 1 \%, leg. Abdinbekova.

\section{Hosts:}

Lycophotia porphyrea (Den. \& SchifF.), Eupsilia transversa (HFN.) (Noctuidae), Lymantria dispar (L.) (Lymantriidae), Operophtera brumata (L.), Agriopis leucophaearia (Den. \& SChIFF.), Agriopis aurantiaria (HвN.), Eupithecia nanata (HвN.) (Geometridae), Thecla betulae (L.) (Lycaenidae), Nola cucullatella(L.) (Nolidae), Poecilocampa populi (L.) (Lasiocampidae), Charaxes jasius L. (Nymphalidae) (HuddLeston 1980), Hypocassida subferruginea Sch. (Chrysomelidae) (Guclu, 2006).

\section{Distribution:}

West Europe, New Zealand, Cyprus, Turkey, Japan (Huddleston, 1980), Turkmenistan, Caucasus, Azerbaijan (Tobias, 1995, Авdinbekova, 1995). 


\section{Meteorus rubens (NeES von ESENBECK, 1811)}

\section{Material:}

dist. Gusar, 7.VIII.1960-62, 3 우 ㅇ, leg. Abdinbekova; Nakhichevan AR, 2.VI.1967, 20 o $^{\circ} 0^{7}$, leg. Abdinbekova; dist. Gobustan, May, 1967, 2 \% $~+$, leg. Abdinbekova.

Hosts:

Agrotis ipsilon (HFN.), Agrotis exclamationis (L.), Mamestra brassicae (L.), Spodoptera exigua (HBN.), Noctua pronuba(L.) (Lepidoptera, Noctuidae), Cynthia cardui (L.) (Lepidoptera, Nymphalidae), Idaea muricata (Hrn.) (Lepidoptera, Geometridae) (Huddleston, 1980).

\section{Distribution:}

Europe, Algeria, Cyprus, Egypt, Israel, Japan, Mongolia, Turkey (Shenefelt 1970), Caucasus, Azerbaijan (Tobias, 1995, Abdinbekova, 1995).

\section{Meteorus versicolor (WESMAEL, 1835)}

\section{Material:}

dist. Khachmaz, 6.VIII.1962-1963, 2 ㅇ ㅇ, leg. Abdinbekova; dist. Guba, 18.VII.1961, 1 ㅇ, leg. Abdinbekova; dist. Kalbajar, 25.VIII.1966, 1 +, leg. Abdinbekova; dist. Goychay, 8.VIII.1964, 3 o $~+$, leg. Abdinbekova; dist. Masally, June,VI.1965, $100^{\top} \sigma^{\star}$, leg. Abdinbekova; dist. Agdash, 22.V.1962, 2 우 ㅇ, leg. Abdinbekova.

Hosts:

Lasiocampa quercus (L.), Malacosoma neustria (L.), Macrothylacia rubi (L.) (Lasiocampidae), Dasychira pudibunda (L.) Euproctis chrysorrhoea(L.), Leucoma salicis (L.), (Lymantriidae), Anarta myrtilli (L.), Lycophotia porphyrea (Den. \& Schif.) (Noctuidae), Eulithis testata (L.) (Geometridae), Maniola jurtina Thaumetopoea processioned (L.) (Satyridae) (HudDLEsTon 1980), Ips typographus L. (Curculionidae) (GucLu, 2006).

\section{Distribution:}

Europe, Mongolia, Turkey (HuddLeston 1980), Caucasus, Azerbaijan (ToBias, 1995).

Genus Microctonus Wesmael, 1835

Microctonus aethiopoides (LOAN, 1975)

\section{Material:}

dist. Guba, 8-26.IV.1962, 4 ○ $^{\star} 0^{\star}$, leg. Abdinbekova; dist. Lankaran, 5.VIII.1964, $10^{7}$, leg. Abdinbekova; dist. Lerik, 30.VI.1965, 1 ơ , leg. Abdinbekova; dist. Ordubad,
5.VI.1967, $4 \bigcirc^{\circledR} \mathrm{o}^{\top}$, leg. Abdinbekova; dist. Norashen (now Sharur), 23.V.1967, 1 ㅇ, leg. Abdinbekova; dist. Mardakert (now Aghdere), 20.VI.1967, $1 \sigma^{\pi}$, leg. Abdinbekova; dist. Julfa, 24.VI.1967, 1 \%, leg. Abdinbekova.

Hosts:

Hypera postica GylL., Hypera meles F., Hypera nigrirostris F., Sitona discoideus Gyll., Sitona lepidus GrLL., Listronotus bonariensis Kusch. (Curculionidae).

\section{Distribution:}

All Palaearctic, North America, Australia and New Zealand, Azerbaijan (ToвiAs, 1986, Авdinвeкоva, 1995).

\section{Microctonus melanopus (RUTHE, 1856 )}

Material:

dist. Ordubad, 2.VI.1967, 2 ㅇ ㅇ , leg. Abdinbekova; Nakhichevan AR, 2.VI.1967, 1 ơ⿱ $^{\dagger}$, leg. Abdinbekova.

Hosts:

Ceutorhynchus assimilis (РАYк.), Ceutorhynchus leprieuri BRIs., Ceutorhynchus obstrictus MARSH., Ceutorhynchus pleurostigma MARsh., Ceutorhynchus quadridens (PAN.), Ceutorhynchus pallidactylus MARsh., Hypera meles (FABR.) (Curculionidae).

\section{Distribution:}

West Europe, Mid Asia, Caucasus, Kazakhstan (ToBias, 1986).

\section{Genus Pygostolus HaLIDAY, 1833}

Pygostolus falcatus (NeEs, 1834)

Material:

dist. Gusar, 10.VII.1967, 1 \%, leg. Abdinbekova; dist. Khachmaz, 28.V.1961, 1 o , leg. Abdinbekova; dist. Mardakert (now Aghdere), 21.VI.1967, 1 o $^{\text {}}$, leg. Abdinbekova.

Hosts:

Brachyderes incanus L., Polydrusus pilosulus CHEvr., Otiorhynchus ovatus L., Strophosoma capitatum DE. GeER., Sitona humeralis STEPH. (Curculionidae), Cryptocephalus bipunctatus L. (Chrysomelidae).

\section{Distribution:}

West Europe, Mongolia, North AmericaKazakhstan, Kirgizia, Siberia, Sakhalin, Caucasus, Azerbaijan (ToBIAS, 1986, Авdinbekova, 1995). 
Genus Syntretus FÖRSTER, 1862

Subgenus Syntretus FöRSTER, 1862

Syntretus (Syntretus) elegans (RUTHE, 1856)

\section{Material:}

dist. Guba, 10.IX.1962, 1 ơ, leg. Abdinbekova; dist. Mardakert (now Aghdere), 28.IV.1967, 2 ㅇ ㅇ, leg. Abdinbekova; dist. Barda, 20.V.1966, 1 ơ, leg. Abdinbekova.

Host:

unknown.

\section{Distribution:}

Europe (VAn Achterberg \& Haeselbarth, 2003), Turkey (Yilmaz, 2008), Far East Russia, Kazakhstan, Georgia, and Lithuania (BelokobylskiJ, 2000), Azerbaijan (AbdinbeKova, 1995).

\section{Genus Wesmaelia FöRSTER, 1862}

\section{Wesmaelia pendula FöRSTER, 1862}

\section{Material:}

dist. Guba, 28.VI.1961, 1 đo leg. Abdinbekova; dist. Goychay, 25.V.1964, $1 \sigma^{\star}$, leg. Abdinbekova.

\section{Hosts:}

Nabis alternatus PARsh., Nabis americolimbatus C., Nabis capsiformis GeRM. (Nabidae).

\section{Distribution:}

Europe (GAvin et al., 2006), Former USSR, Caucasus, Azerbaijan (AbDinbeKova, 1995).

Genus Zele CuRTIS, 1832

Zele chlorophthalmus (SPINOLA, 1808)

\section{Material:}

dist. Guba, 20.VIII.1961, 4 우 우, leg. Abdinbekova; dist. Shahbuz, 14.IX.1967, 10 ๙ $^{\top} 0^{\top}$, leg. Abdinbekova; dist. Gutkashen (now Gabala), 17.VII.1965, $10^{*}$, leg. Abdinbekova; dist. Gakh, 24.VII.1965, $20^{\star \top} 0^{\star}$, leg. Abdinbekova; dist. Shamakhy, 14.VII.1965, $2 \sigma^{\top} \sigma^{\pi}$, leg. Abdinbekova.

\section{Hosts:}

Bupalus piniaria L., Hydriomena furcata ThunB., Rheumaptera hastata L., Semiothisa continuaria Eversm., (Geometridae), Anarta myrtilli (L.) (Noctuidae), Ostrinia nubilalis Hbn., (Pyralidae), Acleris variana Fennald (Tortricidae).

\section{Distribution:}

West Europe, Caucasus, Kazakhstan, Siberia, Azerbaijan (AвDinbeKova, 1995).

\section{Subfamily MACROCENTRINAE}

\author{
Genus Macrocentrus CurTIS, 1833
}

Macrocentrus blandus EADY at CLARK, 1964

\section{Material:}

dist. Dashkasan, 6.VIII.1967, 4 ㅇ 우 , leg. Abdinbekova; dist. Mardakert (now Aghdere), 26.VI.1967, $20^{\top} 0^{\star}$, leg. Abdinbekova; dist. Gadabay, 1.VII.1967, 1 ơ , leg. Abdinbekova.

\section{Hosts:}

Zeiraphera griseana Hвn. (Tortricidae), Agrotis segetum (Den. et Schiff.), Hydraecia petasitis Dвld., Dasypolia templi (Thung.), Mesapamea secalis (L.), Hydraecia micacea (Esp.) (Noctuidae).

\section{Distribution:}

West Europe (GAvin et al. 2011), Turkey (Guclu, 2006), Former USSR, Caucasus, Azerbaijan (АвDINBEKova, 1995).

\section{Macrocentrus collaris (SPINOLA, 1808)}

\section{Material:}

dist. city Sumgayit, 27.IX.1937, 1 \%, leg. Vinovsky; dist.

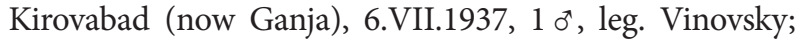
dist. Ordubad, 1-6.VII.1937, 1 \&, $10^{7}$, leg. Vinovsky; dist. Norashen (now Sharur), 13.VI.1959, 6 우 ㅇ, $60^{7} 0^{7}$, leg. Gurbanov; dist. Norashen (now Sharur), 13.VI.1959, $10^{\circ}$,

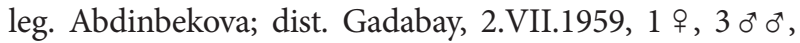
leg. Jafarov; Same locality, 30.VI.1967, 1 \% , 28.VI.1966, 3 우, 28.VIII.1966, 2 우, leg. Abdinbekova; dist. Kurekchay (now in the territory of Goranboy district), 29.VIII.1959, 19 우 , leg. Abdinbekova; dist. Mirbashir (now Tartar), 14-17.VII.1966, 4 우, 1 o, 14. VII.1968, 1 우, leg. Abdinbekova; Same locality, 27.III.1966, 2 우 우, 2 o $^{\star \top}$, leg. Abdinbekova; dist. Barda, 29.VI.1966, 1 o, 1 ㅇ, the same district, village Shirvanly, 17.VI.1966, 1 \% , leg. Abdinbekova; dist. Guba, 22.VII.1962, 1 + , 23.VII.1967,

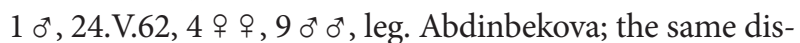
trict village Vladimirovka, 29.VII.1960, 1 đo leg. Abdinbekova; the $11^{\text {th }}$ sovkhoz, 16.V.1961, 1 ㅇ, 29.IX.1961, 2 우,

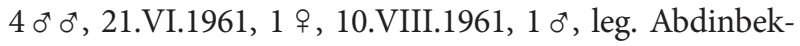
ova; dist. Gusar, 11.VII.1960, 1 o , leg. Abdinbekova; dist. Gasym-Ismayilov (now Goranboy), 18.VI.1966, 1 , leg. Abdinbekova; dist. Norashen (now Sharur), 13.VII.1963, $10^{\star}$, leg. Abdinbekova; dist. Goychay, 2.X.1964, $10^{*}$, leg. Abdinbekova; dist. Masally, 12.V.1965, 2 ㅇ 우, $10^{\circ}$, 
26.VI.1965, 1 o $^{\star}, 16$. VII.1965, 1 우 1 ๙ , leg. Abdinbekova; dist. Lankaran, 6.VI.1965, 3 우 ㅇ, 1 o , 30.VI.1966, 1 , 5.IX.1965, $2 \diamond^{\star} \diamond^{\star}$, leg. Abdinbekova; dist. Ordubad, 7.VII.1965, 1 ㅇ, 1 o , 25.V.1966, 4 ㅇ 우, 2 o $^{\star}$, leg. Mamedov; Same locality, 1.VI.1965, $1 \sigma^{\star}$, leg. Mirzazadeh; Same locality, 3-15.VI.1967, 2 우 ㅇ, 2 đ $0^{\star}$, leg. Abdinbekova; city Baku, 24-25.VII.1960, in the Botanical garden, 1 o , leg. Abdinbekova; dist. Agdash, 13.VI.1966, $1 \sigma^{\star}$, leg. Abdinbekova; dist. Kalbajar, 2.VIII.1966, 1 ơ, 25.VIII.1966, 1 ơ, 9.IX.1966, 8 ㅇ ㅇ , 3 o $^{\star} o^{\star}$, leg. Abdinbekova; dist. Mardakert (now Aghdere), 31.III.1966, 2 우 우 16.VI.1967, 3 우 으, 14.VIII.1966, 3 우 우, leg. Abdinbekova; Nakhichevan AR,

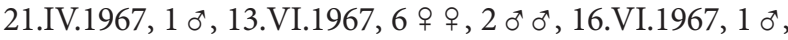
leg. Abdinbekova; dist. Margushevan (now Khojavend), 1.VII.1966, 1 ㅇ , 4.VI.1967, 1 đ, 3 ㅇ , leg. Abdinbekova; dist. Khanlar (now Goygol), 24.VI.1968, 3 , leg. Abdinbekova; dist. Shamakhy, 26.V.1966, 1 \% , the same district, village Chukhuryurd, 27.V.1966, 2 ㅇ ㅇ, leg. Abdinbekova; dist. Agdam, 8.VIII.1966, 2 ㅇ ㅇ, 18.VII.1966, 1 \% , 1 o leg. Abdinbekova; dist. Dashkasan, 2.VIII.1966, 2 우 우 leg. Abdinbekova; Shamkhor (now Shamkir), 19.V.1966, 10 우 우, leg. Abdinbekova; dist. Khankendi, 18.VIII.1966, $10^{\top}$, leg. Abdinbekova; dist. Shahbuz, 14.VI.1967, 2 우 우, $2 \sigma^{\top} \sigma^{\star}$, leg. Abdinbekova; Nakhichevan AR, 2.V.1967, $10^{\star}, 23-28 . V .1967,4$ 우 우 6 o $^{\star}$, leg. Abdinbekova; dist. Julfa, 21.V.1967, 1 o , 3 우 우, leg. Abdinbekova; dist. Zagatala, 17.V.1959, $3 \sigma^{\star} o^{\star}$, leg. Abdinbekova; dist. Masally,
29.III.1965, 1 o , leg. Abdinbekova; dist. Astarkhan-Bazar (now Jalilabad).

Hosts:

Agriotes lineatus L. (Elateridae), Anobium punctatum (Deg.) (Anobiidae), Lycia hirtaria (Cl.) (Geometridae), Polygonia c-album (L.) (Nymphalidae), Eupoecilia ambiguella (HbN.), Den. et Schiff. (Tortricidae), Agrotis clavis (HFn.), Agrotis exclamationis (L.), Agrotis ipsilon (HFN), Agrotis segetum (Den. et ScHIFf.), Euxoa cursoria (HFN), Heliothis armigera (HFN.), Heliothis viriplaca (HFN), Lymantria monacha (L.), Mamestra brassicae (L.), Noctua pronuba (L.), Spodoptera littoralis (BoISD.) (Noctuidae).

\section{Distribution:}

West Europe (GAvin et al. 2011), Turkey (Guclu, 2006), Former USSR, Caucasus, Azerbaijan (AвDINBEKovA, 1995).

\section{Macrocentrus grandii GoIDANICH, 1937}

\section{Material:}

dist. Mirbashir (now Tartar), 12.VI.1966, $20^{\star} o^{\star}$, leg. Abdinbekova; dist. Kalbajar, 25.VIII.1966, 1 ơ, leg. Abdinbekova.

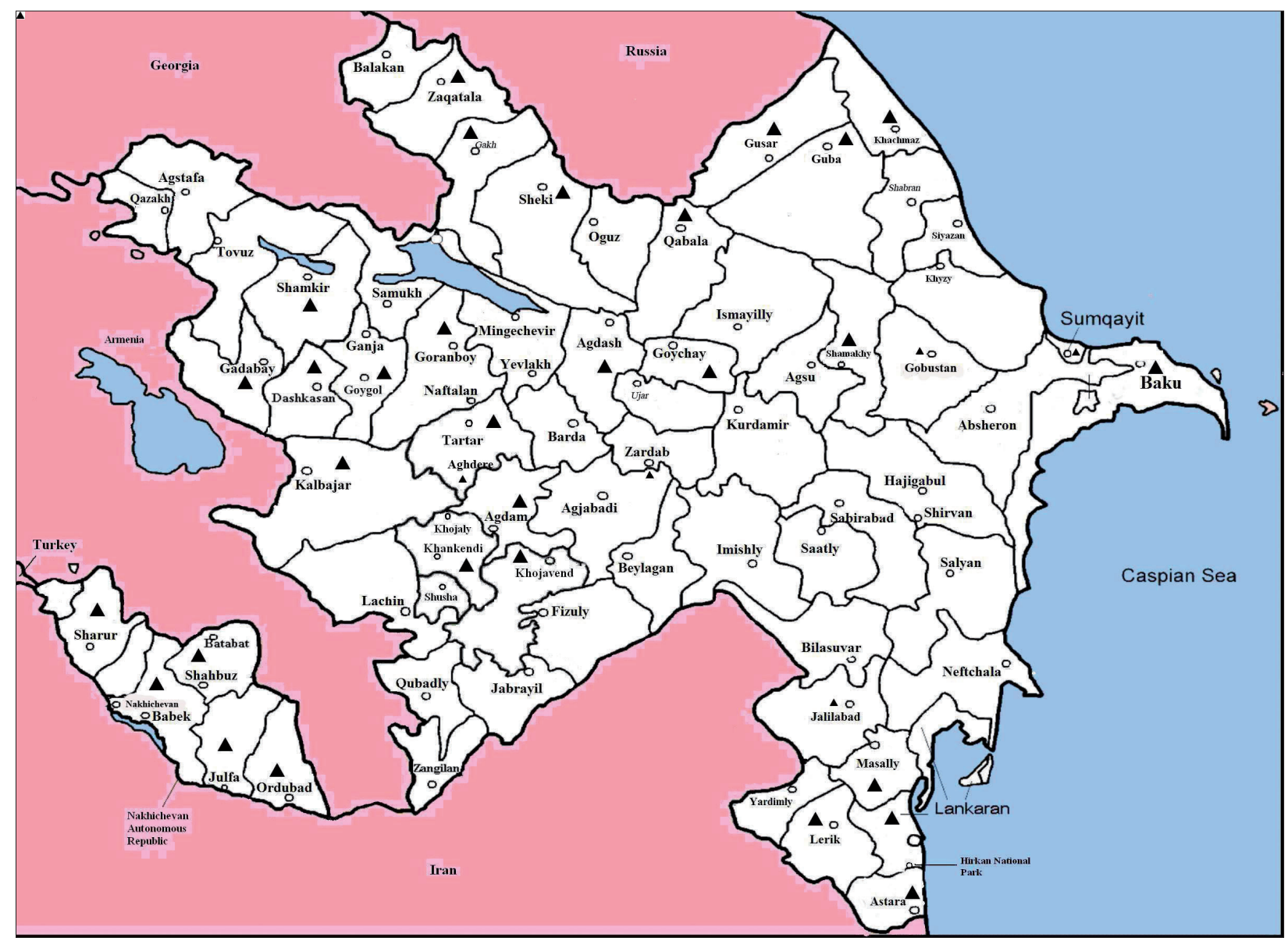

Map 1: Records of Braconidae in Azerbaijan, places (open circles) and localities (filled triangles). 
Hosts:

Vanessa atalanta(L.) (Nymphalidae), Chilo partellus SwIn. (Plutellidae), Eurrhypara hortulata L., Ostrinia furnacalis (GN.) (Pyralidae), Ostrinia nubilalis (HвN.), Pleuroptya ruralis Scop. (Pyralidae).

\section{Distribution:}

West Europe (GAvin et al. 2011), Former USSR, Caucasus, Azerbaijan (AвDinbekova, 1995).

\section{Macrocentrus linearis (NEES, 1811)}

\section{Material:}

dist. Agdam, 21.IV.1960, 1 ㅇ, leg. Abdinbekova; dist. Goychay, 14.V.1964, 1 ơ , leg. Abdinbekova; dist. Guba, 22.VI.1962, 1 ㅇ , leg. Abdinbekova.

\section{Hosts:}

Achlya flavicornis(L.), Drepana arcuata WLK., Drepana falcataria (L.) (Drepanidae), Ennomos quercinaria (HFN.), Ennomos subsignaria (HвN.) (Geometridae), Malacosoma disstria HвN., Malacosoma neustria (L.) (Lasiocampidae), Peribatodes rhomboidaria (DEN. \& SchIF.) (Geometridae), Acleris comariana (LIEN. \& ZELL.), Acleris variegana (DEN. \& Schir.), Aleimma loeflingiana (L.), Archips rosana (L.), Archips xylosteana (L.), Tortrix viridana (L.) (Tortricidae), Acrobasis sodalella Zell., Anania funebris (STr.), Ostrinia nubilalis (HвN.) (Pyralidae), Agonopterix alstromeriana (CL.), Agonopterix nanatella ST. (Depressariidae), Anacampsis populella CL.) (Gelechiidae), Plutella xylostella (L.) (Plutellidae).

\section{Distribution:}

West Europe (GAvin et al. 2011), Former USSR, Caucasus, Azerbaijan (Авdinbeкova, 1995).

\section{References}

Abdinbekova, A. A. 1969: Braconid wasps (Hymenoptera, Braconidae) of Great Caucasus, Azerbaijan (within Azerbaidzhan). - Izvestiya Akademii Nauk Azerbaidzhanskoi SSR. Ser. Biologicheskikh Nauk. 6: 81-91. (in Russian).

Abdinbekova, A. A. 1969: On the knowledge about braconids (Hymenoptera, Braconidae) in the Lankaran zone of Azerbaidjan. - Doklady Akademii Nauk Azerbaidzhanskoi SSR. 25 (9): 59-66. (in Russian).

Abdinbekova, A. A. 1995: Hymenopterans (Hymenoptera, Braconidae) of Azerbaijan. - Baku, 472 p. (in Azery).

Belokobylskij, S. A. 1992: Revision of the genus Centistes Haliday (Hymenoptera: Braconidae: Euphorinae) of the USSR Far East and neighbouring territories. Zoologische Mededelingen 66 (11): 199-237.
Belokobylskij, S. A. 2000: In: P. A. Ler (ed.). Opredelitel nasekomych dalnegobostoka Rossii 4 (4): Vladivostok: 1-650.

Beyarslan, A. 2011: Eubazus (Brachistes) aydae sp. nov. from Turkey (Hymenoptera: Braconidae: Brachistinae). - J. Entomological Research Society, 13 (1): 107-111.

PAPP, J. 2007: Braconidae (Hymenoptera) from Greece, 6 - Notes fauniques de Gembloux 60 (3): 99-127.

PAPP, J. 2009: Braconidae (Hymenoptera) from Mongolia, XVII. Eleven subfamilies. - Acta Zoologica Academiae Scientiarum Hungaricae 55 (2): pp. 139-173.

Huddleston, T. 1980: A revision of the western Palaerctic species of the genus Meteorus (Hymenoptera, Braconidae). - Bulletin of the British Museum Natural History (Entomology) 41: 1-58.

LozAN, A. I. 2004: Braconid wasps from Crete Island (Greece) with description of Chorebus tobiasi sp. n. (Hymenoptera: Braconidae). - Proceedings of Russian Entomological Society, St. Petersburg, Vol. 75 (1): 122-126

Shenefelt, R. D. 1970: Braconidae 11. Introduction. Guide to Host Names Index to Braconid Name. - In: Hymenopterum Catalogus 16: 1-384.

Tobias, V. I.; Belokobylskij, S. A. \& Kotenko, A. G. 1995: Keys to the Insects of the European Part of the USSR: Volume III Hymenoptera Part IV: 883 pp. (in Russian).

Yilmaz, T.; AydoĞdu, M. \& Beyarslan, A. 2010: The distribution of Euphorinae wasps (Hymenoptera: Braconidae) in Turkey, with phytogeographical notes. - Turkish Journal of Zoology 34: 181-194.

VAN ACHTERBerg, C. 1988: Revision of The subfamily Blacinae Foerster (Hymenoptera, Braconidae). Zoologische Verhan.: pp. 249-324.

Van Achterberg, C. \& Haeselbarth, E. 2003: Revision of the genus Syntretus Foerster (Hymenoptera: Braconidae: Euphorinae) from Europe Zoologische Mededelingen 77 (2): 9-78.

Vladimir, Ž.; VAN Achterberg, K. \& Stankovici, S. 2010: A contribution to Braconidae, Hybrizontidae (Hymenoptera: Ichneumonoidea) and Stephanidae (Hymenoptera: Stephanoidea) from the SouthWest Balkans. - Acta Entomologica Serbica, 15 (2): 227-235. 